PDFlib PLOP: PDF Linearization, Optimization, Protection

Page inserted by evaluation version www.pdflib.com - sales@pdflib.com 


\title{
Sensory Responses to 6-n-Propylthiouracil (PROP) or Sucrose Solutions and Food Preferences in Young Women ${ }^{a}$
}

\author{
ADAM DREWNOWSKI ${ }^{b}$ SUSAN AHLSTROM HENDERSON, \\ AMY B. SHORE, AND ANNE BARRATT-FORNELL \\ Human Nutrition Program, School of Public Health, The University of Michigan, Ann \\ Arbor, Michigan 48109-2029, USA
}

\begin{abstract}
Genetic sensitivity to the bitter taste of 6-n-propylthiouracil (PROP) has been linked with a greater number of food aversions and reported rejection of some bitter foods. Healthy young women $(n=121)$ were divided into nontasters, tasters and supertasters of PROP according to their PROP detection thresholds and the ratio of intensity ratings of $P R O P$ versus $\mathrm{NaCl}$ solutions. Hedonic response profiles to sucrose solutions distinguished between likers and dislikers of sweet. All subjects completed a 171-item food preference checklist. Food preference data were reduced by factor analyses, subscales of which were tested for reliability using Cronbach's alpha. Greater PROP sensitivity was associated with lower acceptance of coffee, cruciferous vegetables, tart citrus fruit, dark breads, and selected fats. In contrast, liking for sucrose solutions was linked to liking for sugar in tea and coffee, but not to any special pattern of food acceptance. Strategies aimed at increasing the consumption of grains, vegetables, and fruit should consider the role of inherited taste markers and their potential impact on dietary habits.
\end{abstract}

\section{INTRODUCTION}

Dietary strategies for the prevention of chronic disease typically aim to increase the consumption of grains, vegetables, and fruits. ${ }^{1,2}$ To date, nutrition education and intervention programs have been based on social and psychological models of behavioral change. ${ }^{3.4}$ In contrast, the role of taste responsiveness as a potential barrier to the adoption of healthy eating habits has received less research attention. ${ }^{5}$

Yet most consumers state that it is taste that determines their food choices. ${ }^{6}$ While many factors can shape food preferences, sensory factors are the key reason for food rejection. Bitter taste, in particular, has been cited as the main reason for disliking coffee, alcohol, some cheeses, bitter cruciferous vegetables and some citrus fruit. ${ }^{7-10}$ Low acceptance of bitter-tasting vegetables and fruits may prevent some consumers from adopting healthy diets consistent with dietary guidelines.

Some taste responses and food dislikes may be genetically determined ${ }^{5,8}$ Sensitivity to the bitter taste of phenylthiocarbamide (PTC) and 6-n-propylthiouracil (PROP) is a heritable trait. ${ }^{11,12}$ Though past studies tried to link PTC/PROP sensitivity with a pattern of food dislikes, no consistent results were observed. Food preference scores can

\footnotetext{
"Supported by Grant CA 61680.

" Please address all correspondence to: Adam Drewnowski, Ph.D., Human Nutrition Program, SPH II, 1420 Washington Heights, Ann Arbor, MI 48109-2029. Tel: (313) 765-3277; fax: (313) 7635455; cmail: adamdrew@umich.edu
} 
be highly variable, especially across sexes, ages, and a broad range of dieting habits and ethnic and cultural backgrounds.

\section{METHODS}

Subjects were 121 healthy women nonsmokers, mean age $27.7 \mathrm{yr}$ (range 20-60 yr) and mean body mass index (BMI) $23.3 \mathrm{~kg} / \mathrm{m}^{2}$. PROP detection thresholds were determined by the method of solutions as described previously. ${ }^{7}$ Subjects also tasted 5 PROP solutions containing $0.000032,0.0001,0.00032,0.001$, and $0.0032 \mathrm{~mol} / \mathrm{L} \mathrm{PROP}$ (solutions $7,9,11,13$, and 15$) ; \mathrm{NaCl}$ solutions $(0.01$ to $1.0 \mathrm{~mol} / \mathrm{L} \mathrm{NaCl})$, and 5 sucrose solutions $(0.058$ to $0.93 \mathrm{~mol} / \mathrm{L}$ sucrose $)$. Using previously described procedures, we identified 37 nontasters, 49 regular tasters, and 35 supertasters of PROP. The distribution of taste thresholds is shown in Figure 1.

Subjects with ascending hedonic functions for sucrose solutions were defined as likers, while those with descending hedonic functions were defined as sucrose dislikers. ${ }^{13,14}$ This procedure yielded 50 likers and 63 dislikers

Subjects completed a 171-item food preference checklist. Subjects were asked to indicate how much they liked or disliked each food using a standard 9-point hedonic preference scale. Factor analyses were used to reduce the data for individual foods $(n=171)$ into a smaller number of food aggregates. Separate factor analyses were conducted for vegetables, fruits, grains, meats, desserts, beverages, fats and sweetener categories. Principal components factor analysis with varimax rotation generally yielded solutions of between one and three factors per category that accounted for between $47 \%$ and $66 \%$

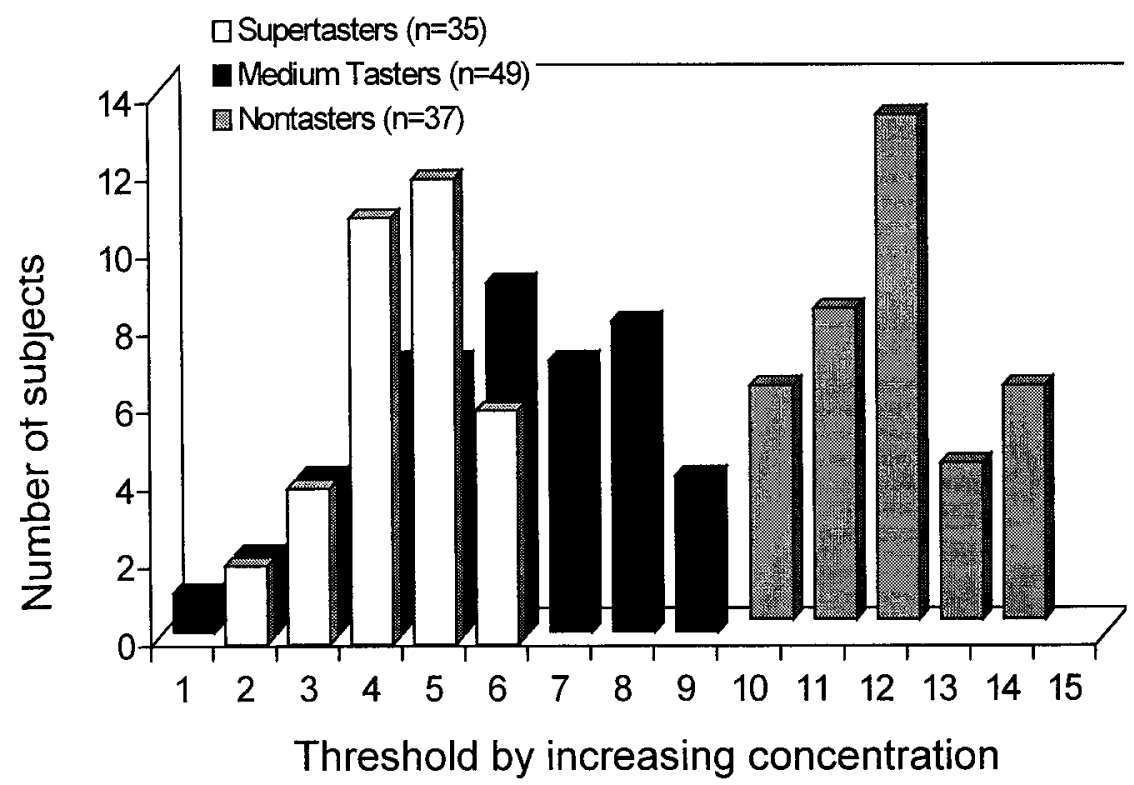

FIGURE 1. Distribution of taste detection thresholds for 6- $n$-propylthiouracil (PROP) by taster status. 
of the variance. Subscales constructed on the basis of factor analyses were then tested for reliability using Cronbach's alpha. Differences in mean subscale scores between nontasters and supertasters of PROP, and dislikers and likers of sweet, were then tested for significance using $t$-tests for independent samples.

\section{RESULTS}

As shown previously, supertasters rated PROP solutions as more bitter than did either tasters or nontasters and disliked PROP solutions more than did either tasters or nontasters.

Mean preference ratings for vegetable and fruit categories are shown in TABLE 1. PROP sensitivity was associated with lower acceptance of cruciferous vegetables (Brussels sprouts, cauliflower, raw and cooked cabbage, and radishes). The relationship between PROP sensitivity and dislikes was linear, with supertasters showing lower ratings than did either regular tasters or nontasters.

PROP sensitivity was also associated with lower acceptance of bitter citrus and tart fruits (grapefruit, grapefruit juice, lemons, rhubarb). Again supertasters disliked bitter citrus fruits the most. Another significant contrast between nontasters and supertasters was observed in acceptance ratings for soft fruits and berries. No other differences were statistically significant.

In contrast to PROP data, sucrose likers gave higher ratings to sugar in coffee and tea, and higher ratings on the pie subscale (apple, pumpkin and sweet potato) than did dislikers. No other significant differences were observed.

\section{DISCUSSION}

PROP sensitivity was associated with greater dislike of coffee beverages, whole grain breads, bitter cruciferous vegetables, and bitter and sour fruits. Consistent with previous data, ${ }^{8,15}$ significant differences were obtained for food subscales that included Brussels sprouts, coffee, dark breads and grapefruit juice. Whereas preferences (scores $>5$ ) were not necessarily associated with PROP taster status, subjects who disliked these foods were generally sensitive to the bitter taste of PROP. For coffee, dark breads and cereals, reported dislike was associated with sharply lower reported frequencies of consumption.

In cases where PROP sensitivity was associated with greater dislikes, the relationship between the two was linear. The fact that supertasters of PROP disliked coffee, grapefruit juice and other foods more than did either tasters or nontasters supports the validity of the taster/supertaster distinction. Given the substantial proportion of supertasters in the female population (estimated by Bartoshuk at $25 \%$ ), the potential impact of taste responses on diet habits of women can have substantial implications for women's health.

The present data suggest that PROP sensitivity does not affect food acceptance across the entire range of response options. Foods that are highly preferred may be liked for reasons other than taste, and PROP sensitivity may only have a minor impact. On the other hand, food dislikes and food rejections are likely to be strongly influenced by taste factors, and low acceptance ratings for vegetables and fruits were linked to PROP sensitivity. A major aim of behavioral strategies for the adoption of healthy diet is to increase preferences for nutrient-dense but marginally liked foods. As a result, genetic taste factors are likely to play a major role in determining the success of nutrition education and intervention programs. 


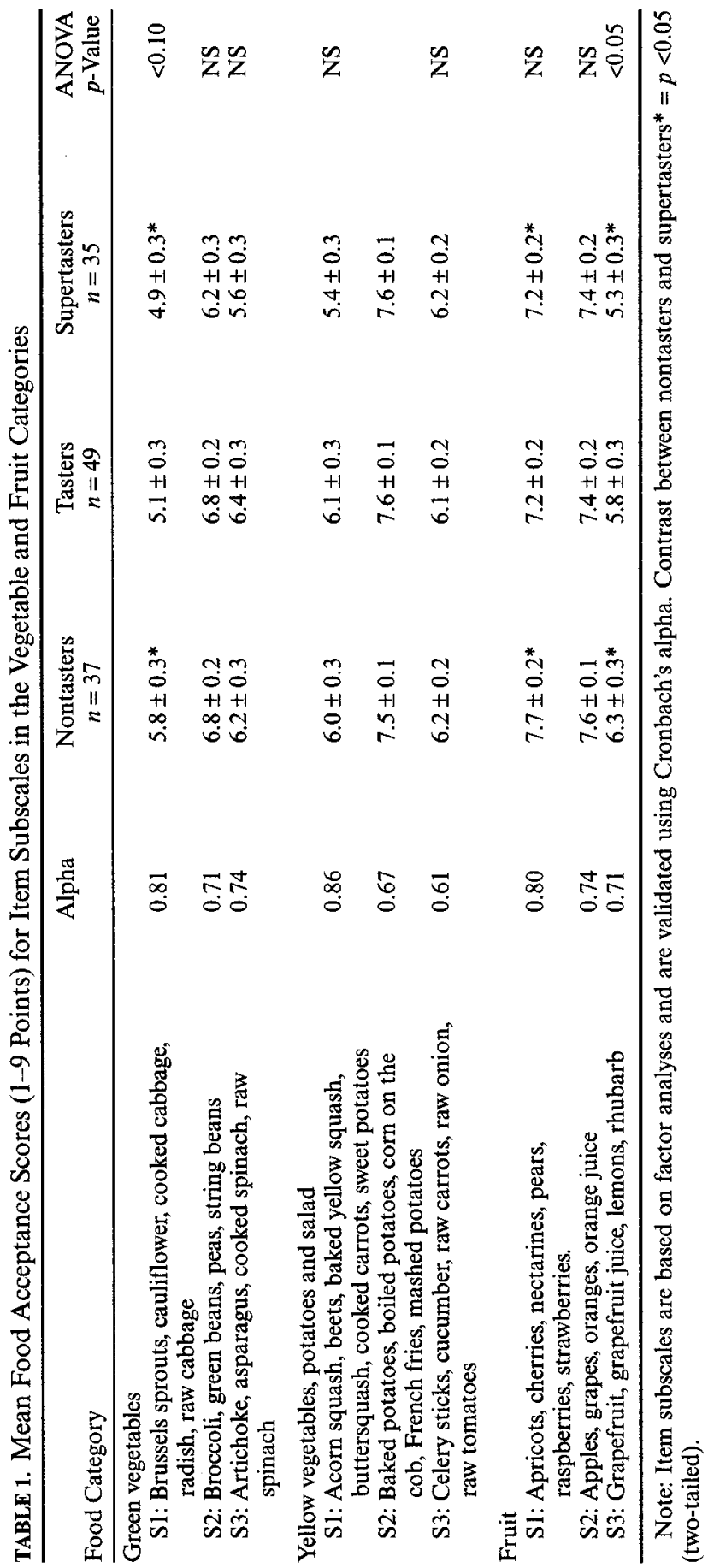




\section{REFERENCES}

1. Healthy People 2000: National Health Promotion and Disease Prevention Objectives. 1991. DHHS Publication No. (PHS) 91-50213. US Department of Health and Human Services, Public Health Service. Washington, DC.

2. Kennedy, E., L. Meyers \& W. LAyden. 1996. The 1995 dietary guidelines for Americans: An overview. J. Am. Diet. Assoc. 96: 234-237.

3. Glanz, K., F. M. Lewis \& B. K. Rimer, Eds. 1990. Health Behavior and Health Education: Theory, Research, and Practice. Jossey-Bass. San Francisco.

4. Havas, S., et al. 1994. 5 a day for better health: A new research initiative. J. Am. Diet. Assoc. 94: $32-36$.

5. Drewnowski, A., S. A. Henderson \& A. B. Shore. 1997. Taste responses to naringin, a flavoroid, and the acceptance of grapefruit juice are related to genetic sensitivity to 6- $n$ propylthiouracil (PROP). Am. J. Clin. Nutr. 66: 391-397.

6. Drewnowski, A. 1997. Taste preferences and food intake. Annu. Rev. Nutr. 17: 237-253.

7. Bartoshuk, L. M. 1993. The biological basis of food perception and acceptance. Food Qual. Pref. 4: 21-32.

8. Drewnowski, A. \& C. L. Rock. 1995. The influence of genetic taste markers on food acceptance. Am. J. Clin. Nutr. 62: 506-511.

9. Glanville, E. V. \& A. R. Kaplan. 1965. Food preference and sensitivity of taste for bitter compounds. Nature 205: 851-853.

10. KRONDL, M. et al. 1983. A twin study examining the genetic influence on food selection. Hum. Nutr. Appl. Nutr. 37A: 189-198.

11. Fox, A. F. 1932. The relationship between chemical constitution and taste. Proc. Natl. Acad. Sci. USA 18: 115-120.

12. Kalmus, H. 1971. Genetics of taste. In Handbook of Sensory Physiology. L. M. Beidler, Ed.: 165-179. Springer-Verlag. Berlin.

13. Drewnowski, A. \& M. Schwartz. 1990. Invisible fats: Sensory assessment of sugar/fat mixtures. Appetite 14: 203-217.

14. Looy, H. \& H. P. Weingarten. 1992. Facial expressions and genetic sensitivity to 6- $n$ propylthiouracil predict hedonic response to sweet. Physiol. Behav. 52: 75-82.

15. Forrai, G. \& G. Bankovi. 1984. Taste perception for phenylthiocarbamide and food choice-A Hungarian twin study. Acta Physiol. Hung. 64: 33-40 